\title{
COMMUNICATION
}

\section{Tuberculose extra pulmonaire cervico faciale}

Tabeti-Bentahar CF${ }^{1}$, Bouzouina $\mathrm{F}^{1}$, Metahri $\mathbf{M}^{2}$

1 - Service de Pathologie et Chirurgie Buccales CHU Oran Algérie

2 - Service de Pneumologie CHU Oran Algérie

La tuberculose, maladie à déclaration obligatoire, est une infection bactérienne chronique due au Mycobacterium Tuberculosis (bacille de Koch). La tuberculose peut être pulmonaire ou extrapulmonaire, tels les cas rapportés. Le $1^{\text {er }}$ cas : un garçon de 06 ans, présentant à l'examen clinique une asthénie et une dysphagie avec une tuméfaction submandibulaire gauche, et des caries des canines et des molaires temporaires. L'orthopantomogramme a révélé une 75 à l'état de racines. Le diagnostic de cellulite submandibulaire gauche est retenu. La 75 est extraite sous antibiothérapie. Le patient est perdu de vue puis revu après 07 mois avec persistance de la tuméfaction. L'échographie cervicale a montré des polyadénopathies ; l'interrogatoire a retrouvé une notion de fièvre nocturne. L'intradermoréaction à la tuberculine (IDRt) était phlycténulaire de $16 \mathrm{~mm}$. Le $2^{\text {ème }}$ cas : une fillette de 08 ans présentant à l'examen clinique un état général altéré avec fièvre, une tuméfaction submandibulaire droite mal limitée avec signe du godet positif et des caries des 54, 74, 75, 84 et 85 . L'IDRt était phlycténulaire de $14 \mathrm{~mm}$. L'échographie cervicale a révélé un magma d'adénopathies latéro-cervicales bilatérales. La cytoponction a conclu à une adénite tuberculeuse en activité. Le $3^{\text {ème }}$ cas : une femme de 34 ans, hospitalisée en gynéco-obstétrique pour grossesse précieuse de 8 mois avec abcès dentaire. L'examen clinique a retrouvé une tuméfaction submandibulaire gauche dure et indolore. L'orthopantomogramme a révélé une réaction apicale au niveau de la 38 dont l'extraction s'est faite sous antibiothérapie par voie intraveineuse. La malade est revue 2 mois plus tard avec reprise des signes infectieux. Une tomodensitométrie a objectivé une lyse osseuse du ramus gauche. La ponction et la biopsie ont permis de poser le diagnostic de tuberculose. Le $4{ }^{\text {ème }}$ cas : une femme de 30 ans, présentant des éruptions nodulaires violacées au niveau du nez et du sillon nasolabial droits évoluant depuis un an associées à une ulcération palatine. L'IDRt était phlycténulaire de $22 \mathrm{~mm}$. Les biopsies de l'aile du nez et du palais ont confirmé le diagnostic de tuberculose cutanéo-muqueuse. Chez les 4 patients, le téléthorax et la sérologie ont permis d'éliminer respectivement une localisation pulmonaire et une atteinte par le HIV. La prise en charge thérapeutique des 4 malades s'est faite au service de Pneumologie où un traitement antituberculeux a été instauré avec rémission totale des lésions.

Le diagnostic positif de tuberculose repose sur l'examen histologique, les cultures et les tests cutanés tuberculeux. La radiographie pulmonaire confirme l'atteinte pulmonaire (Laskaris 2007). En 2013, selon les estimations, 9 millions de personnes l'ont contractée et 1,5 million de personnes en sont décédées, dont 360000 sujets VIH-positifs (OMS 2014). La tuberculose est le plus souvent pulmonaire et environ 1/3 des cas sont à localisation extrapulmonaire (Coulon et Piette 
2008). Paradoxalement, en Algérie, en 2014, sur un total de 22517 nouveaux cas déclarés, 8809 cas étaient pulmonaires et 13708 cas extra pulmonaires (OMS 2014). La tuberculose primaire de la muqueuse buccale est rare et elle est souvent secondaire à une atteinte pulmonaire (Coulon et Piette 2008) mais ce diagnostic doit être évoqué devant toute ulcération buccale. La tuberculose des maxillaires peut simuler une cellulite d'origine dentaire comme dans les trois premiers cas et l'odontostomatologiste peut être confronté à un réel défi diagnostique et donc la demande d'un téléthorax et d'une IDRt doit être systématique au moindre doute. 